\title{
Developmental changes in reward processing are reward specific
}

\section{Authors}

Sebastijan Veselic ${ }^{2,3^{*}}$, Claire Rosalie Smid ${ }^{1}$, Nikolaus Steinbeis ${ }^{1}$

*corresponding author (sebastijan.veselic.18@ucl.ac.uk)

${ }^{1}$ Division of Psychology and Language Sciences, University College London, UK

${ }^{2}$ Clinical and Movement Neurosciences, Department of Motor Neuroscience, University College

London, London, UK

${ }^{3}$ Wellcome Centre for Human Neuroimaging, University College London, London, UK 


\section{Abstract}

Rewards have a profound impact on human motivation, cognition, affect and behavior. The study of reward processing and the effects of incentives thus occupies a central place in psychology and cognitive neuroscience. A core assumption when comparing groups or individuals is that reward types are valued equally. Here we test the validity of this assumption in a sample of 26 adults 23 children (7-11 years) using both primary (i.e. pleasant taste) and secondary rewards (i.e. money). We show that adults value money more than pleasant tastes, while children value them equally, a developmental pattern replicated across three distinct tasks including subjective valuation, willingness to exert cognitive effort, and the invigorating effects on cognitive control. Our findings suggest that money increases in value with age, presumably with experience, and warrant caution when interpreting group differences in reward processing, at least in a developmental framework. Future work should expand to using incentives that are appropriate to the groups under investigation.

\section{Keywords}

Value-based decision making, subjective value, development of value, motivation, cognitive control, cognitive effort 


\section{Introduction}

Humans, like other species, are highly sensitive to rewards ${ }^{1,2}$. One primary function of rewards is their capacity to energize and motivate actions directed towards achieving specific goals. Rewards thus play a pivotal role in shaping human motivation, cognition, affect and behavior, impacting processes as diverse as learning, decision making, and cognitive control ${ }^{3-5}$. As such, the study of reward processing and its influence on behavior occupies a central field of research in psychology and cognitive neuroscience. One key assumption in research on reward processing is that the subjective value of the studied reward is invariant across individuals or groups of individuals studied. This assumption is particularly salient in developmental psychology, where the majority of studies assume that the specific incentives are of comparable value to all, and that potential group differences are due to differences in the underlying processing systems rather than difference in the subjective value assigned to specific rewards (see also ${ }^{6,7}$. Here we test whether this assumption holds by comparing processing of two reward types (i.e. primary and secondary) between groups of children and adults across a range of different experimental paradigms and show that it does not. Instead, reward processing and its impact on motivation and cognition for each group is strongly reward specific.

The development of reward processing and the effects of rewards on motivation, cognition, affect and behavior have been studied and documented extensively, highlighting considerable differences between children, adolescents, and adults. This includes age-related changes in phenomena as wide-ranging as reward sensitivity ${ }^{8}$, risk-taking ${ }^{9,10}$, intertemporal choice ${ }^{11-13}$, prosocial behavior ${ }^{14-16}$, model-based decision-making ${ }^{17}$, reward exploration ${ }^{18}$, and cognitive control 19. Such observable developmental differences in behavior are implicitly linked to maturational changes in reward-related brain regions. For instance, evidence of changes in dopamine receptor (D1R and D2R) density in striatum and prefrontal cortex ${ }^{20-23}$, changes in resting state connectivity of striatal regions ${ }^{24}$, as well as in corticostriatal connectivity from childhood into adulthood ${ }^{25-29}$. While there is little doubt that reward processing does indeed change with age (e.g. ${ }^{30,31}$, true effects may have been falsely estimated due to the overwhelming use of money as the primary incentive (but for exceptions see Galvan et al., ${ }^{32-34}$. Money is a 
cultural artefact, the value of which is presumably acquired only with experience ${ }^{35,36}$, it is highly probable that value of money increases with age. Alternatively, the novelty of money might make this reward type particularly desirable for younger age groups ${ }^{37}$. Irrespective of both accounts, the fact that monetary rewards may not be valued stably across development ${ }^{7}$, risks an inaccurate estimation of genuine developmental trajectories in reward processing. This is especially relevant given that the goals humans pursue change as a function of development ${ }^{38}$. As a consequence, differences in goals being pursued also constrain the types of rewards we value and how subjectively valuable we find them to be ${ }^{7}$.

Here we directly assess the effects of different reward types by comparing the effects of primary (i.e. pleasant tastes) and secondary (i.e. money) rewards on children's and adults' 1) explicit assignment of subjective value, 2) their willingness to exert cognitive effort, and 3) their allocation of cognitive control. We compared primary rewards with secondary rewards, given that preferences for pleasant tastes are established prenatally, remain relatively stable through development ${ }^{39}$ and are arguably less contaminated by the experience-dependent acquisition of value compared to secondary rewards ${ }^{40}$. Responses to primary rewards are therefore likely to be more informative of genuine developmental change in reward processing. We show that compared to pleasant taste, adults value money more, that they are more willing to exert cognitive effort for money, and that money has a stronger invigorating effect on cognitive control. In contrast, across all three paradigms, the two reward types were virtually indistinguishable for children. 


\section{Results}

\section{a Explicit valuation}

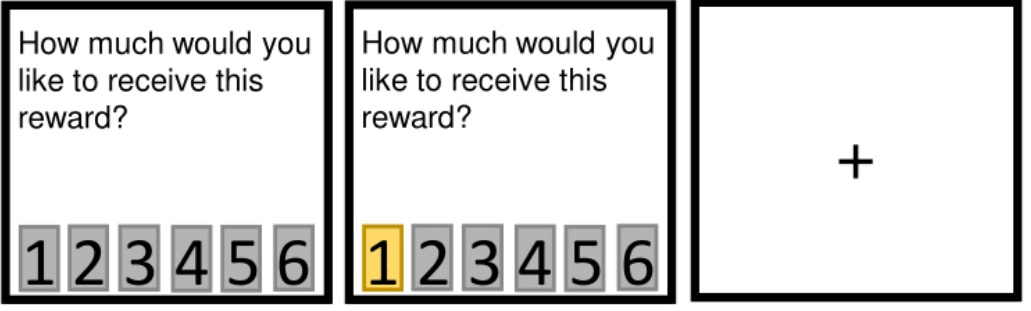

\section{b Willingness to work}

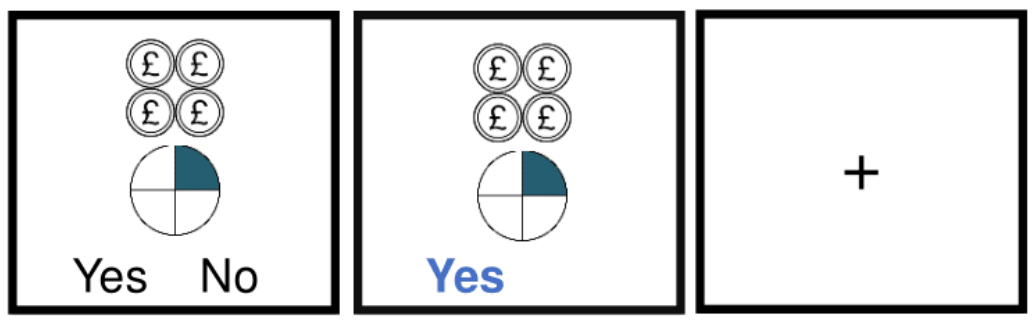

\section{c Cognitive control}
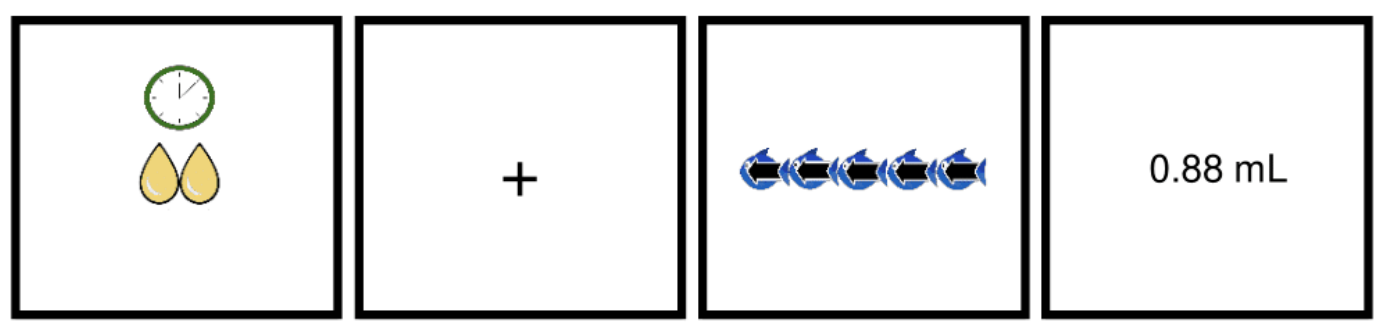

Figure 1. Study and task description.

\section{Reward-specific valuation changes with development}

To test our main hypothesis, we first assessed whether participants' subjective valuation of different reward types interacted with age (Figure 1a). We found an interaction (Figure 2a) between group and reward type $\left(F_{(1,91)}=4.96, p=0.03\right)$. Adults preferred secondary $(M=5.15+-0.248)$ over primary $(M=4.28+-0.21)$ rewards $\left(t_{25}=2.78,95 \% \mathrm{Cl}[0.226,1.52], p=0.01\right)$ whereas children $\operatorname{did} \operatorname{not}\left(M=4.72(0.249), M=4.49(0.31) ;\left(t_{22}=0.62,95 \% \mathrm{Cl}[-1.01,0.55], p=0.54\right)\right.$. The observed developmental interaction was not explained by groups differences in their perception of how much juice is worth in pounds $\left(t_{44.27}=1.12, p=0.26\right.$, Figure $\left.2 b\right)$, with the interaction remaining significant when this information was added as a covariate. Furthermore, the observed developmental 
interaction remained significant when we investigated further covariates, such as how thirsty participants were in the beginning of the experiment (for further controls see Supplementary Materials).
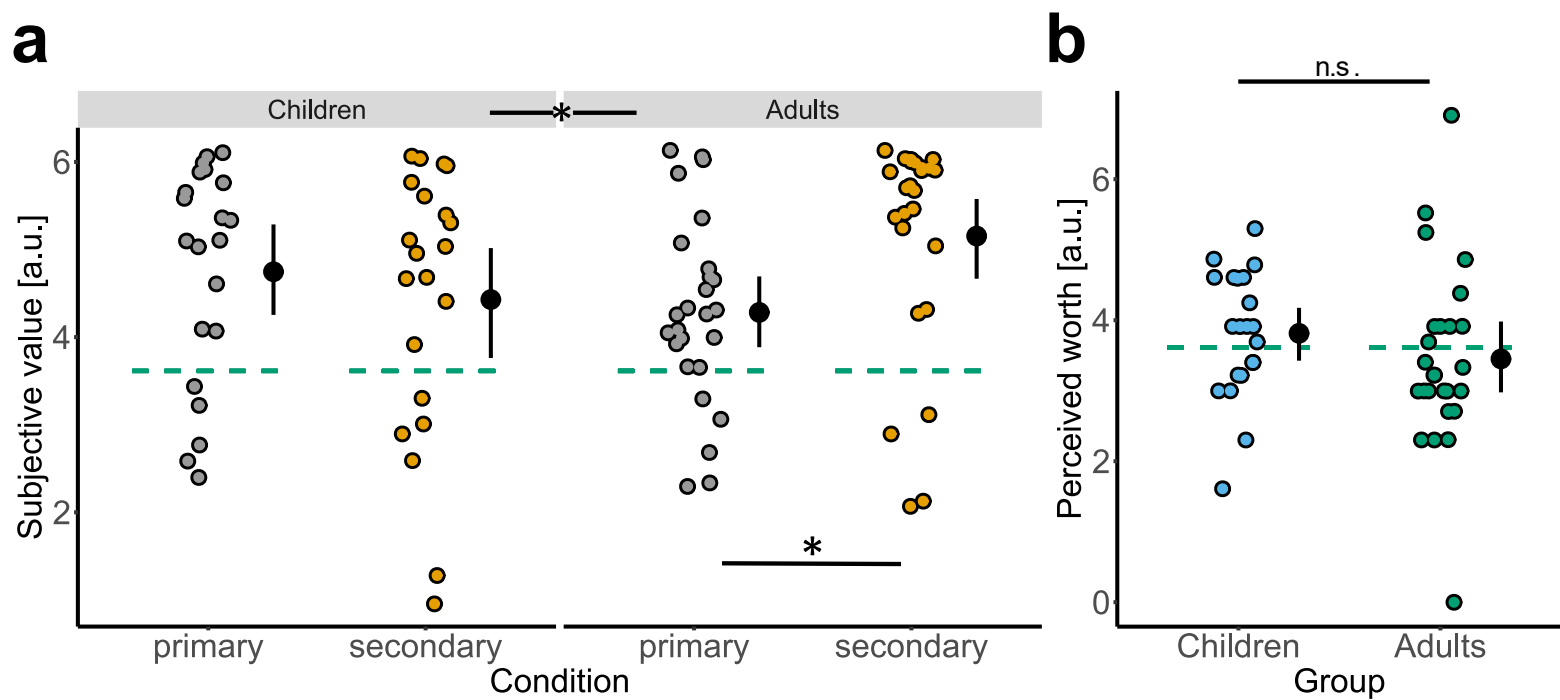

Figure 2. Subjective value of reward changes across development.

\section{Developmental changes in the effect of rewards on cognitive effort are reward- specific}

Next, we investigated whether participants' differences in reward-specific valuation was reflected in their willingness to exert cognitive effort for such rewards (for full task description see Methods and Supplementary Materials). We used a novel and age-adapted cognitive effort task where we parametrically varied reward size and difficulty level of a cognitively effortful task for both primary and secondary rewards and investigated how this modulated participants' willingness to exert cognitive effort (Figure 1b).

We first observed that participants' discounted the value of rewards as a function of cognitive difficulty (Figure 3a), similar to previous work in physical ${ }^{41}$, cognitive ${ }^{42,43}$ or time ${ }^{44}$ discounting. To quantify and statistically evaluate the behavioral group differences in Figure $3 \mathrm{a}$, we fit three different computational models that have been previously used in the effort and time-discounting literature (hyperbolic, exponential, effort-sensitive ${ }^{1}{ }^{43-45}$ and compared the discounting rate

\footnotetext{
${ }^{1}$ We used the term effort-sensitive to denote the adaptation of the time-sensitive model to varying levels of cognitive effort as opposed to varying levels of time delays.
} 
parameter estimates across both groups and conditions (for details on model selection see Supplementary Materials). There was a significant interaction between group and reward for the discounting rate $(\mathrm{k})$ parameter of the hyperbolic model which measures how strongly rewards are discounted as a function of cognitive effort that needs to be exerted to attain them $\left(F_{(1,92)}=9.09\right.$, $p<.01$, Figure $3 b)$. Adults discounted value more strongly for the primary compared to secondary reinforcer $\left(t_{25}=2.24,95 \% \mathrm{Cl}[0.004,0.11], p=0.03\right)$. However, this difference was reversed in children who discounted value more strongly for the secondary compared to the primary reinforcer $\left(t_{21}=6.96,95 \% \mathrm{Cl}[0.08,0.14], p<0.001\right)$. Finally, adults more strongly discounted value in the primary conditions compared to children $\left(t_{35.642}=3.9,95 \% \mathrm{Cl}[0.075,0.24], p=0.0004\right)$. These results demonstrate that adults and children had reversed preferences about what they were willing to exert cognitive effort for.

As a control, participants were required to rate the experienced cognitive load corresponding to individual cognitive difficulty levels they were making choices about in the willingness to work task (Figure 1b). To do this, participants were required to solve items of the matrix reasoning subtest at each difficulty level at the end of the experiment. Both groups rated more difficult items as cognitively more effortful (children: $F_{(3,79)}=9.97, p<.001$; adults: $F_{(3,91)}=2.9, p=0.04$, Figure 3c). 

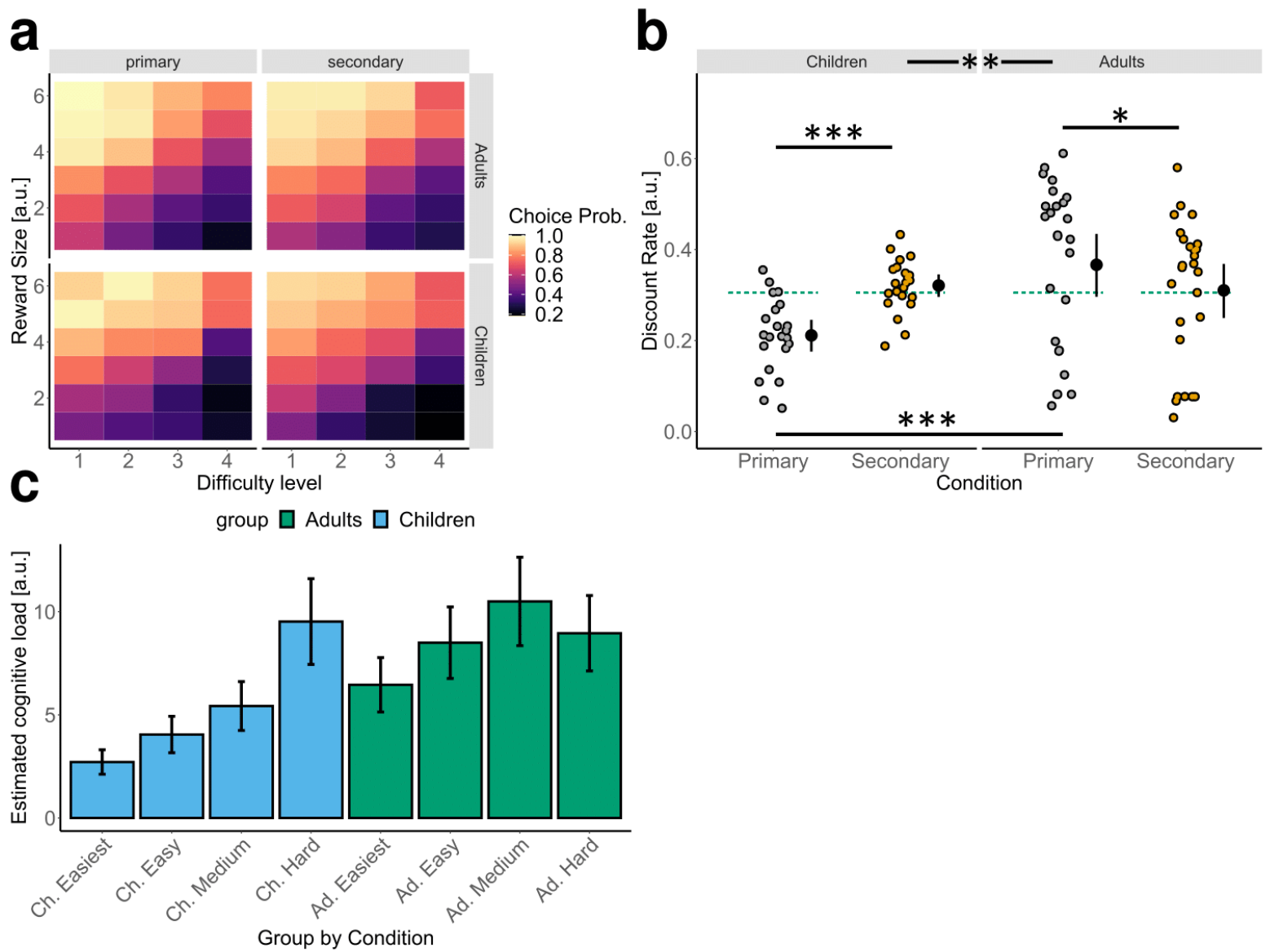

Figure 3. Reward specific effects on exertion of cognitive effort across development.

\section{Developmental changes in the invigorating effect of rewards on cognitive control are reward-specific}

Finally, we looked at reward-specific effects on cognitive control (Figure 1c) and how these might interact with development. We modelled participants' responses on a trial by trial basis using generalized linear mixed modelling and hypothesised that the degree to which cognitive control is exerted on a go-no-go task will be modulated by reward ${ }^{19,27,46-48}$, and that the degree to which it is exerted as a function of reward would mirror our results on the previous two tasks. We observed a similar interaction as in the other two tasks where adults performed better on go trials when rewarded with secondary compared to primary rewards $\left(t_{25}=4.73, p<.001\right)$, while children's performance was comparable across both reward types (Figure $4 a, \chi 2=8.11, p=0.004$; see also Supplementary Materials Table S1). Considering we observed similar interactions between group and reward type on go trials of the cognitive control task and in the subjective valuation task, we 
predicted the self-reported subjective value would be correlated with the results from Figure 4a. Indeed, we observed that adults' subjective value of secondary rewards was correlated with interindividual differences in the go trials for secondary $(r=0.51, p=0.008)$ but not primary rewards $(r=0.26, p=0.19)$. In children, these correlations had a reversed sign but were not significant for either the primary $(r=-22, p=0.22)$ or secondary $(r=-0.21, p=0.35)$ reward. Despite this correlation, we observed no correlation with the k-value parameters from the previous task where subjects were required to exert cognitive effort (all $p>.161$ ). This means our results could not be driven simply due to e.g. more attentive subjects having better performance across all the tasks or similar related confounds.

Finally, we investigated whether a developmental interaction with reward-type would also be observed in no-go trials, requiring participants to inhibit their prepotent response. We observed a trending interaction $(\chi 2=2.91, \mathrm{p}=0.085)$ mirroring the finding on go-trials (see Supplementary Materials Table S3) where adult performance was improved on trials with a secondary relative to primary reinforcer, however this was not significant $\left(t_{25}=1.89, p=0.07\right)$. That is, adults were marginally better at inhibiting the prepotent response for secondary compared to primary reinforcers while children performed equally well. 

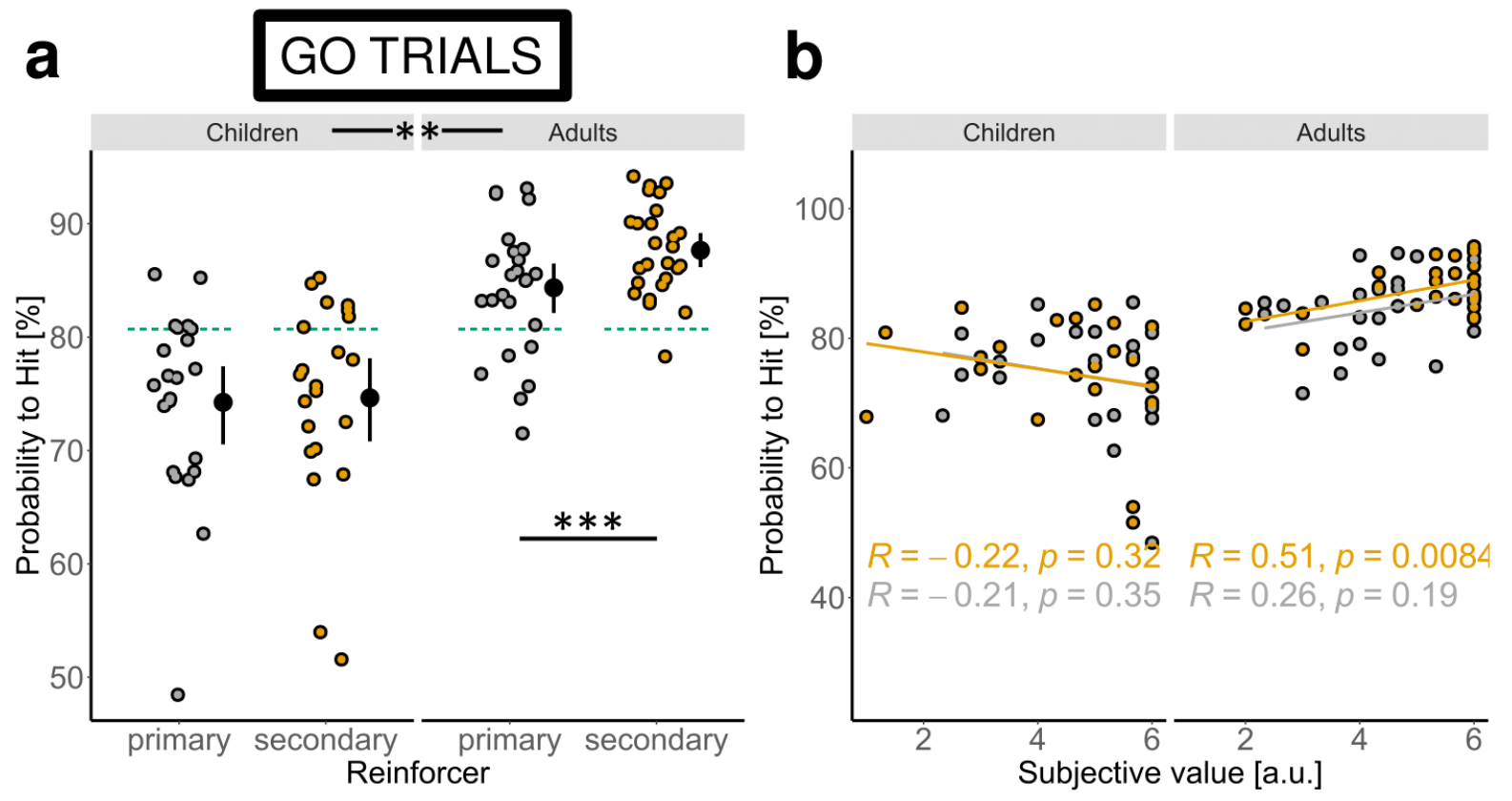

\section{NO-GO TRIALS}

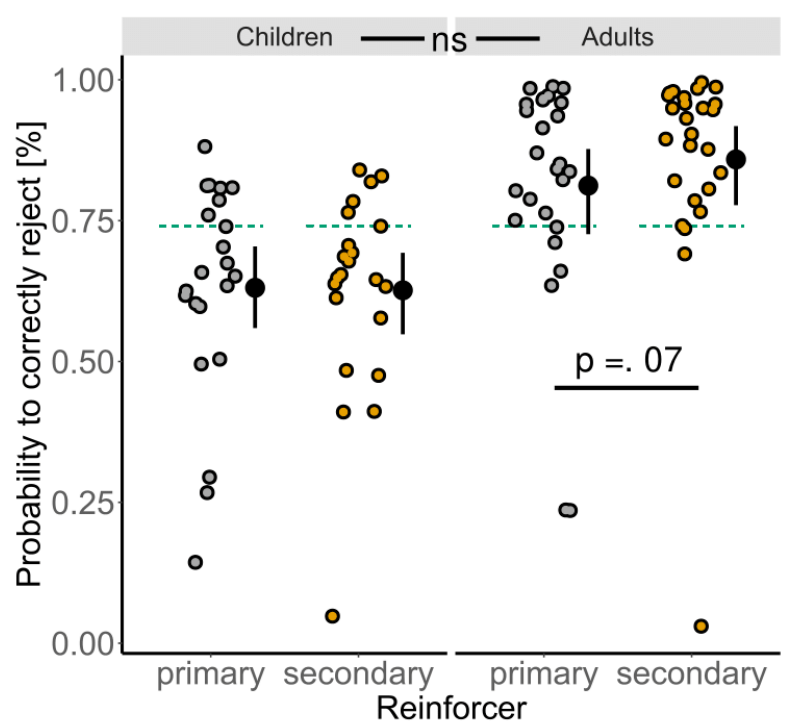

Figure 4. Cognitive control is invigorated in a reward-specific way across development. 


\section{Discussion}

Secondary rewards such as money (or vouchers) are some of the most prominently used incentives to study the development of a range of cognitive, motivational, and socio-affective functions $11,12,16,17,19,27,42,49-54$. Thus, it has been found that compared to adults, children or adolescents take greater monetary risks ${ }^{53,54}$, are less generous ${ }^{51}$, and more impatient ${ }^{16,52}$, while at the same time being less affected by money in boosting their executive functions ${ }^{27,49}$. These findings are interpreted on the basis that the incentives used have the same value across developmental populations. However this assumption may be highly problematic considering that the value with money is presumably highly experience-dependent ${ }^{36}$. Here we use both primary and secondary rewards (e.g. pleasant tastes and money respectively), to compare whether it is the rewarding properties of money or reward processing per se that changes with age. If developmental change is generic to reward processing, then we would expect similar findings for both reward types. However, across three different experimental paradigms, our findings show that money is valued more by adults than by children. This decided preference was present in explicit statements, a willingness to work, and the invigoration effects on cognitive control, confirming our central hypothesis that rewards are valued differently by children and adults ${ }^{7}$.

These findings suggest a revision of current interpretations of developmental change across swaths of psychology and cognitive neuroscience and call into question the prevalent use of money to study the development of a range of cognitive, motivational, and socio affective functions $11,12,16,17,19,27,42,49-54$. At the very least, these results urge for more concerted efforts to design experimental paradigms that employ genuinely developmentally appropriate stimuli. Furthermore, they imply the development of reward processing in similar tasks ${ }^{55}$ may have been misrepresented due to differences in subjective value. Note, while, our study does not provide a parsimonious explanation for previous findings, it highlights the necessity of cautiously interpreting developmental patterns when using money, whatever these may be.

What mechanism might drive the observable preference of money in adults? We assume this might be experience considering the prevalence of different rewards changes through development, and 
this is especially true for money compared to pleasant tastes. For example, adults work for money and consider it to be the main medium through which other goods can be attained, where this may not necessarily be true in children. This crucial difference between both groups is related to the notion that the type of goals children pursue in their daily life is likely a key factor dictating what kind of rewards children perceive as valuable compared to adults ${ }^{7,38}$. More formally, these results can be directly tied to reinforcement learning theory ${ }^{2}$ which predicts money would acquire value throughout childhood and adolescence into adulthood as we learn that acquiring money is predictive of rewarding outcomes (e.g. being able to purchase ice-cream or a house), while no such learning necessarily occurs with juice. This is corroborated to a certain degree by previous work showing adolescents aged 12 and above already are invigorated by money when it comes to exertion of physical effort ${ }^{56}$.

Our results suggest rewards used to study the differences in reward processing between children and adults need to be better operationalized and developed more adequately. One way of achieving this is to consider what is ecologically relevant to the niche either adults or children inhabit. For example, children pursue different goals to adults (e.g. children do not work to attain money with which to pay rent, bills, etc.). Therefore, a more careful design of developmentally appropriate incentives in developmental research will enable a more precise study of the effect of rewards on cognition across development, as considered in ${ }^{30,31}$. It is worth noting there are clear and genuine changes taking place in reward processing across development, as evidenced by a developing reward circuitry from childhood to adulthood ${ }^{25,26,29}$. However, the extent of these developmental differences can only be meaningfully gaged if we take into account that behavioral and neural responses to incentives will be reward-specific. Here we argue that behavioral changes particularly linked to reward needs to be understood within the ecology of the developing individual 57.

In conclusion, our results show processing of rewards across development is reward specific when it comes to subjective value, willingness to control, and the invigorating effect rewards have on cognitive control. Our results question the prevalent use of monetary incentives to study 
developmental changes in reward processing. We argue for a more thoughtful and careful operationalisation of incentives that are more relevant to the studied samples.

\section{Acknowledgements}

We would like to thank Somya lqbal, Joshua Spowage, and Harriet Phillips for their help with organizing the data collection of the child sample. We would like to thank Marie Mueller and Roser Canigueral for their feedback on a previous version of the manuscript. SV was supported by the Leverhulme Award DS-2017-026. CRS was supported by the European Research Council (European Research Council (ERC) grant agreement no. 715282, project DEVBRAINTRAIN). NS was supported by the European Research Council (European Research Council (ERC) grant agreement no. 715282, project DEVBRAINTRAIN) and a Jacobs Research Fellowship.

\section{Author Contributions}

SV and NS designed the experiment. SV and CRS collected the data. SV analysed the data. SV wrote the manuscript. SV, CRS, and NS edited the final manuscript.

\section{Competing interests}

No competing interests declared. 


\section{Methods}

\section{Participants}

Twenty-six adults (13 male, age $=24.61$ years, range $=18.7-35.3$ years) were recruited though university listings. Twenty-three children ( 14 male, age $=9.4$ years, range $=7.1-10.85$ years) from schools in the Greater London area. One child was excluded from all analyses due to failing to provide most responses. Participants were pre-screened for juice-related allergies. Ethical approval for this study was obtained from our university's research ethics committee in compliance with national regulations, project ID number: 12271/003.

\section{Procedure}

Adults were required to avoid drinking any fluids for 4 hours before the experiment to ensure adequate thirst levels ${ }^{58,59}$. This was not done for the children participants due to ethical reasons, however we did control for thirst levels by asking participants at the beginning of the experiment how thirsty they are to be able to investigate whether thirst levels account for their subjective valuation (see Supplementary Materials). At the end of the experiment we also collected data indicating when was the last time they had consumed any liquids. The experiment was divided in three sections.

First, we collected information about participants' preferences for different juices flavours (e.g. pineapple, apple, orange), which they could select for the main experiment. We additionally gathered information on their preferences about the go-no-go stimuli which would be presented to them during the cognitive control task (fish stimuli) by asking how much they like them on a scale of 1-6. Finally, this was also repeated for their thirst and tiredness levels. Furthermore, we administered the matrix reasoning subtest of the Wechsler Abbreviated Scale of Intelligence II. This test was administered because it is standardized, meaning the perception of cognitive difficulty in the willingness to work task could be compared across all participants in a robust and statistically meaningful way. 
Next, participants performed three tasks under two reward type conditions (juice and money). These tasks measured explicit valuation of rewards, willingness to work for different rewards, and cognitive control. Task sequence and reward type were counterbalanced across participants. Each participant received automated on-screen instructions throughout these tasks and an experimenter was present to ensure the instructions were understood equally across participants. Before doing these tasks, participants were introduced to both reward types. Both groups were told primary rewards would involve receiving varying amounts of their favourite juice. For secondary rewards, adults were told their rewards would be translated into pounds after the experiment, while children were told they would be receiving monetary points they would later be able to exchange for possible rewards ranging in attractiveness. These rewards were not shown to them to ensure that differences across participants in how much they liked them would not influence their motivation. For both groups, the visual stimuli they saw related to rewards were identical.

After the three main tasks, participants were required to make choices on trials probing their willingness to work for rewards. They were also required to answer additional questions and questionnaires which we used to control for confounds. These involved a need for cognition scale, answering about their perceived worth of $20 \mathrm{ml}$ of juice in pounds, and a control task estimating the cognitive effort participants reported when solving items of specific difficulty levels from the Wechsler Abbreviated Scale of Intelligence II.

\section{Tasks}

Explicit valuation: In this task participants were shown either a picture of a juice carton indicating primary reward or a pound coin indicating secondary reward. Crucially, children were told that the pound coin corresponds to the points they will be able to earn and exchange for a reward and not actual money. Participants were asked to provide answers to three questions on a scale from 1 (least) to 6 (most): "How pleasant do you find this reward? How much would you like to receive this reward? How happy would you feel right now about this reward?' These questions were modelled on previous work in the field ${ }^{60}$. 
Willingness to work: We developed a novel task for measuring willingness to work modelled on previous work studying temporal ${ }^{61}$ and cognitive discounting of value ${ }^{42,62}$. Participants were required to make binary yes/no choices whether they were willing to exert varying levels of cognitive effort (4 levels) to obtain a reward varying in size (6 levels) and type (primary, secondary reward). The trial combinations were repeated three times in pseudorandom order for a total of 144 trials with an additional 4 trials as familiarization in the beginning of the task. Participants' decisions were not timed and the stimuli remained on the screen until they made their choice. They received a prompt after 2 seconds to make a choice. During the instruction phase they were explicitly told what the individual difficulty levels corresponded in terms of their experience when they were solving items on the matrix reasoning subtest from the first section.

A critical component of the willingness to work task was that subjective experience of effort for the four difficulty levels they were making choices about is as comparable as possible across participants and within each age group. We approximated this by grounding the subjective experience of difficulty for the willingness to work task in the matrix reasoning subtest. The advantage of doing this is that the matrix reasoning subtest is standardized on a large population and individual differences in performance can be measured in t-score distances within each group. As a result, differences in "easiest", "easy", "medium", "hard" were measured in t-score distances that were constant across participants based on their performance.

All participants confirmed they understood the distinction between the four difficulty levels and could recall from their experience individual items from the matrix reasoning subtest that would correspond to those difficulty levels. The task instruction was to decide whether they were willing to exert a certain amount of cognitive effort for a given reward level. From all choices, two choices (one for each reward type) would be randomly selected towards the end of the experiment. If a trial where they pressed "no" would be selected, they would forfeit any chance to obtain additional rewards. If a trial where they pressed "yes" would be selected, they would get the reward if they would provide a correct answer to it. If they would provide an incorrect answer, there was still a low probability (20\%) that they would obtain the reward. This low probability was added to optimally 
incentivise participants to choose "yes" even on more difficult trials (see Supplementary materials for a more detailed explanation).

To ensure participants perceived the different difficulty levels as cognitively more effortful, they were subject to a control task. Participants saw stimuli denoting the upcoming difficulty level (easiest, easy, medium, hard) followed by an item from the matrix reasoning subtest of that difficulty level, titrated to their individual performance on that subtest. They were given unlimited time to solve the individual items and after each answer they were required to provide answers on the NASA Task Load Index ${ }^{63}$. We used the cognitive load question as an indicator of exerted cognitive effort. Importantly, while we used this control task to explicitly monitor whether more difficult items from the matrix reasoning subtest corresponded to more exerted cognitive effort, participants actually never saw the mapping between difficulty level and item during the willingness to work task described above. This was done to keep their experience of individual difficulty levels uncontaminated by the control task. That is, the control task was only used for validating that participants are able to distinguish their exerted cognitive effort across the difficulty levels of the matrix reasoning subtest.

Cognitive control: We used a standard go-no-go task as used previously (Brydges et al., 2013; Bunge. et al., 2002) with an instruction procedure that was modelled after ${ }^{58}$. Participants were required to press "f" or " $k$ " depending on the direction of a visual stimulus in the shape of a fish (left $-f$, right $-k$ ) or omit an answer depending on the color (red - omit response on $33 \%$ trials, blue press on $66 \%$ trials). The task was structured as a combination of a flanker task with only congruent trials and a go-no-go task. In total, there were three main factors: reward (primary, secondary), difficulty (easy, difficult), and reward size (low, high). Each response was followed by an outcome that depended on the reward type condition and reward size condition. For secondary rewards, adults would see an increment in pounds for correct responses (2 pence for low, 6 pence for high reward size) while children would correspondingly see the same but were explicitly instructed these were points that would be later exchanged for a reward of a particular size, as described in the procedure. For primary rewards, we used a gustatory stimulation device that was connected to the 
go-no-go task. On trials with a correct response, participants would receive a squirt of juice that depended on the reward size $(0.02 \mathrm{ml}$ for low and $0.06 \mathrm{ml}$ for high reward size). Notably, the visual stimuli used for high and low reward size differed for from the visual stimulus indicating reinforcer type. However, we believe this difference is unlikely to account for the observed results. Participants initially had a period of 10 trials for familiarization and establishing their reaction times that were then used as baseline for how much time they had to provide a response on easy and difficult trials. On each trial they initially saw the condition combination (difficulty and reward size). Then the stimulus was presented for $300 \mathrm{~ms}$. Across the easy and hard conditions, they had 400 and 200 ms cut-offs to provide a response, respectively. The mean reaction time was continuously updated by computing the reaction time for the last ten trials for each condition on a rolling basis. This ensured differences across adults or children in loss of motivation or tiredness could not explain differences in performance on this task. After their response, they would see the feedback for $1000 \mathrm{~ms}$. Randomized trial sequences were generated for each participant and in total there were approximately 550 trials per participant, with 30 trials on average per individual condition.

\section{Analysis}

Explicit valuation: we used a general linear model to investigate whether there was a group by reward type interaction on participants' subjective value. The subjective value was the average of answers participants gave in this task. We used counterbalancing order as a nuisance regressor to control for the order in which this question occurred (e.g. for some participants it occurred after they had already performed the cognitive control task and knew how well they performed with a particular reward type). We additionally ran a control model where we added further nuisance regressors with questions related to thirst (how strongly they desire, crave, and how thirsty they are) and perceived monetary worth of juice, as mentioned in the supplementary materials.

Willingness to work: We fitted three different discounting models previously used in the literature; see e.g. ${ }^{45,61}$ - using hierarchical Bayesian model fitting in JAGS ( Ahn et al., 2017). These models were (hyperbolic): 


$$
S V=\frac{R}{1+k T}
$$

Where SV denotes subjective value, $\mathrm{R}$ denote reward, $\mathrm{k}$ denotes the discounting rate, $\mathrm{T}$ denotes the cognitive difficulty of a trial. Cognitive difficulty was measured in t-scores as individual items were titrated such that, across participants any pair of differences (easy to medium or medium to high) had a constant t-score difference for a population of normative participants. The further two models were the exponential (top) and effort sensitivity (bottom) model:

$$
\begin{gathered}
S V=R e^{-k T} \\
S V=R e^{-r t^{S}}
\end{gathered}
$$

Where $r$ is the effort discounting parameter, and $s$ is the effort sensitivity parameter. We estimated the winning model using leave one out criterion ( $\mathrm{LOOIC},{ }^{67}$ ) and used the maximum a posteriori estimates of the parameter distribution to test for a group by reward type interaction in the parameter estimates using GLMs.

We further used a GLM to test for a main effect of difficulty in both groups in the control task while controlling for the items they were presented with in the control task. This was done to establish that the individual difficulty levels we used in the willingness to work task were actually perceived as requiring more cognitive effort by participants. Namely, the actual mapping between difficulty and item was never shown to participants explicitly prior to this control task. Therefore, their responses on the willingness to work task were uncontaminated by their mapping to individual items on the matrix reasoning subtest and required an explicit control test.

Cognitive control: We used linear mixed effects modelling to investigate our hypothesis that a group by reward type interaction would predict participants' probability of providing a correct response on the go-no-go task. We investigated this in two separate models, one fitted on go trials 
and the other fitted on no-go trials to distinguish between being able to provide a correct response by initiating an action and being able to provide a "correct" response by being able to inhibit a prepotent response. In the results, we report participant level model coefficients from the winning model. In the supplementary results, we show the full results of the winning models in individual tables. 


\section{References}

1. Dayan, P. \& Niv, Y. Reinforcement learning: The Good, The Bad and The Ugly. Curr. Opin. Neurobiol. 18, 185-196 (2008).

2. Lee, D., Seo, H. \& Jung, M. W. Neural Basis of Reinforcement Learning and Decision Making. Annu. Rev. Neurosci. 35, 287-308 (2012).

3. Rangel, A., Camerer, C. \& Montague, P. R. A framework for studying the neurobiology of value-based decision making. Nat. Rev. Neurosci. 9, 545-556 (2008).

4. Daeyeol, L., Seo, H. \& Jung, M. W. Neural Basis of Reinforcement Learning and Decision Making. Annu. Rev. Neurosci. 35, 287-308 (2012).

5. O'Doherty, J. P., Cockburn, J. \& Pauli, W. M. Learning, Reward, and Decision Making. Annu. Rev. Psychol. 68, 73-100 (2017).

6. Kray, J., Schmitt, H., Lorenz, C. \& Ferdinand, N. K. The influence of different kinds of incentives on decision-making and cognitive control in adolescent development: A review of behavioral and neuroscientific studies. Front. Psychol. 9, 1-21 (2018).

7. Davidow, J. Y., Insel, C. \& Somerville, L. H. Adolescent Development of Value-Guided Goal Pursuit. Trends Cogn. Sci. 22, 725-736 (2018).

8. Van Duijvenvoorde, A. C. K. et al. Neural Correlates of Expected Risks and Returns in Risky Choice Across Development. J. Neurosci. 35, 1549-1560 (2015).

9. Galvan, A., Hare, T., Voss, H., Glover, G. \& Casey, B. J. Risk-taking and the adolescent brain: Who is at risk? Dev. Sci. 10, 8-14 (2007).

10. Braams, B. R., van Duijvenvoorde, A. C. K., Peper, J. S. \& Crone, E. A. Longitudinal changes in adolescent risk-taking: A comprehensive study of neural responses to rewards, pubertal development, and risk-taking behavior. J. Neurosci. 35, 7226-7238 (2015).

11. Van Den Bos, W., Rodriguez, C. A., Schweitzer, J. B. \& McClure, S. M. Adolescent impatience decreases with increased frontostriatal connectivity. Proc. Natl. Acad. Sci. U. S. A. 112, E3765-E3774 (2015).

12. de Water, E., Cillessen, A. H. N. \& Scheres, A. Distinct age-related differences in temporal discounting and risk taking in adolescents and young adults. Child Dev. 85, 1881-1897 (2014).

13. Green, L., Fry, A. F. \& Myerson, J. Discounting of delayed rewards: A Life-Span Comparison. Psychol. Sci. 5, 33-36 (1994).

14. Blake, P. R. \& Rand, D. G. Currency value moderates equity preference among young 
children. Evol. Hum. Behav. 31, 210-218 (2010).

15. Fehr, E., Bernhard, H. \& Rockenbach, B. Egalitarianism in young children. Nature 454, 10791083 (2008).

16. Steinbeis, N., Haushofer, J., Fehr, E. \& Singer, T. Development of Behavioral Control and Associated vmPFC-DLPFC Connectivity Explains Children's Increased Resistance to Temptation in Intertemporal Choice. Cereb. Cortex 26, 32-42 (2016).

17. Decker, J. H., Otto, A. R., Daw, N. D. \& Hartley, C. A. From Creatures of Habit to GoalDirected Learners: Tracking the Developmental Emergence of Model-Based Reinforcement Learning. Psychol. Sci. 27, (2016).

18. Schulz, E., Wu, C. M., Ruggeri, A. \& Meder, B. Searching for rewards like a child means less generalization and more directed exploration. bioRxiv 327593 (2018) doi:10.1101/327593.

19. Hardin, M. G., Schroth, E., Pine, D. S. \& Ernst, M. Incentive-related modulation of cognitive control in healthy, anxious, and depressed adolescents: development and psychopathology related differences. J Child Psychol Psychiatry 48, 446-454 (2007).

20. Andersen, S. L., Thompson, A. P., Krenzel, E. \& Teicher, M. H. Pubertal changes in gonadal hormones do not underlie adolescent dopamine receptor overproduction.

Psychoneuroendocrinology 27, 683-691 (2002).

21. Seeman, P. et al. Human brain dopamine receptors in children and aging adults. Synapse 1, 399-404 (1987).

22. Tarazi, F. I., Tomasini, E. C. \& Baldessarini, R. J. Postnatal development of dopamine D4-like receptors in rat forebrain regions: Comparison with D2-like receptors. Dev. Brain Res. 110, 227-233 (1998).

23. Simon, N. W. \& Moghaddam, B. Neural processing of reward in adolescent rodents. Dev. Cogn. Neurosci. 11, 145-154 (2015).

24. Fareri, D. S. et al. Normative development of ventral striatal resting state connectivity in humans. Neuroimage 118, 422-437 (2015).

25. Asato, M. R., Terwilliger, R., Woo, J. \& Luna, B. White matter development in adolescence: A DTI study. Cereb. Cortex 20, 2122-2131 (2010).

26. Gogtay, N. et al. Dynamic mapping of human cortical development during childhood through early adulthood. Proc. Natl. Acad. Sci. U. S. A. 101, 8174-8179 (2004).

27. Insel, C., Kastman, E. K., Glenn, C. R. \& Somerville, L. H. Development of corticostriatal 
connectivity constrains goal-directed behavior during adolescence. Nat. Commun. 8, (2017).

28. Barnea-Goraly, N. et al. White matter development during childhood and adolescence: A cross-sectional diffusion tensor imaging study. Cereb. Cortex 15, 1848-1854 (2005).

29. Caballero, A., Granberg, R. \& Tseng, K. Mechanisms contributing to Prefrontal Cortex Maturation during Adolescence. Neurosci. Biobehav. Rev. 70, 4-12 (2016).

30. Williams, J., Ashill, N. \& Thirkell, P. How is value perceived by children? J. Bus. Res. 69, 5875-5885 (2016).

31. Gelman, S. A. \& Echelbarger, M. E. Children, Object Value, and Persuasion. J. Consum. Psychol. 29, 309-327 (2019).

32. Csikszentmihalyi, M., Larson, R. \& Prescott, S. The Ecology of Adolescent Activity and Experience. J. Youth Adolesc. 6, 281-294 (1977).

33. Blair, J. \& Lacy, M. G. Risky Business: The Paradoxical MEaning of Problem Behavior for Young Adolescents. Maggs, Jennifer Almeida, David Galambos, Nancy 15, 344-362 (1995).

34. Guyer, A. E., McClure-Tone, E. B., Shiffrin, N. D., Pine, D. S. \& Nelson, E. E. Probing the neural correlates of anticipated peer evaluation in adolescence. Child Dev. 80, 1000-1015 (2009).

35. Searle, J. The Construction of Social Reality. Philosophy 71, 313-315 (1995).

36. Brandstiitter, E. \& Brandstiitter, H. What's money worth? Determinants of the subjective value of money. J. Econ. Psychol. 17, 443-464 (1996).

37. Bevins, R. A. \& Besheer, J. Novelty reward as a measure of anhedonia. 29, 707-714 (2005)

38. Hofferth, S. \& Sandberg, J. How American Children Spend Their Time. J. Marriage Fam. 63, 295-308 (2001).

39. Mennella, J. A. \& Bobowski, N. K. The sweetness and bitterness of childhood: Insights from basic research on taste preferences. 152, 502-507 (2015).

40. Ventura, A. K. \& Worobey, J. Early influences on the development of food preferences. Curr. Biol. 23, R401-R408 (2013).

41. Bonnelle, V., Manohar, S., Behrens, T. \& Husain, M. Individual Differences in Premotor Brain Systems Underlie Behavioral Apathy. Cereb. Cortex 26, 807-819 (2016).

42. Chevalier, N. Willing to Think Hard? The Subjective Value of Cognitive Effort in Children. Child Dev. 89, 1283-1295 (2018). 
43. Chong, T. T. J. et al. Neurocomputational mechanisms underlying subjective valuation of effort costs. PLoS Biol. 15, 1-28 (2017).

44. Johnson, M. W. \& Bickel, W. K. Within-subject comparison of real and hypothetical money rewards in delay discounting. J. Exp. Anal. Behav. 77, 129-146 (2002).

45. Ebert, J. E. J. \& Prelec, D. The fragility of time: Time-insensitivity and valuation of the near and far future. Manage. Sci. 53, 1423-1438 (2007).

46. Jazbec, S. et al. Age-related influence of contingencies on a saccade task. Exp Brain Res 174, 754-762 (2006).

47. Padmanabhan, A., Geier, C. F., Ordaz, S. J., Teslovich, T. \& Luna, B. Developmental changes in brain function underlying the influence of reward processing on inhibitory control. Dev. Cogn. Neurosci. 1, 517-529 (2011).

48. Geier, C. F., Terwilliger, R., Teslovich, T., Velanova, K. \& Luna, B. Immaturities in reward processing and its influence on inhibitory control in adolescence. Cereb. Cortex 20,16131629 (2010).

49. Niebaum, J. C., Chevalier, N., Guild, R. M. \& Munakata, Y. Adaptive control and the avoidance of cognitive control demands across development. Neuropsychologia 123, 152-158 (2019).

50. Schulz, E. \& Gershman, S. J. The algorithmic architecture of exploration in the human brain. Curr. Opin. Neurobiol. 55, 7-14 (2019).

51. Steinbeis, N., Bernhardt, B. C. \& Singer, T. Impulse Control and Underlying Functions of the Left DLPFC Mediate Age-Related and Age-Independent Individual Differences in Strategic Social Behavior. Neuron 73, 1040-1051 (2012).

52. Achterberg, M., Peper, J. S., van Duijvenvoorde, A. C. K., Mandl, R. C. W. \& Crone, E. A. Frontostriatal white matter integrity predicts development of delay of gratification: A longitudinal study. J. Neurosci. 36, 1954-1961 (2016).

53. Defoe, I. N., Dubas, J. S., Figner, B. \& Van Aken, M. A. G. A meta-analysis on age differences in risky decision making: Adolescents versus children and adults. Psychol. Bull. 141, 48-84 (2015).

54. Levin, I. P., Hart, S. S., Weller, J. A. \& Harshman, L. A. Stability of choices in a risky decisionmaking task: A 3-year longitudinal study with children and adults. J. Behav. Decis. Mak. 20, 241-252 (2007).

55. Strang, N. M. \& Pollak, S. D. Developmental continuity in reward-related enhancement of cognitive control. Dev. Cogn. Neurosci. 10, 34-43 (2014). 
56. Rodman, A. M. et al. How adolescents and adults translate motivational value to action: Agerelated shifts in strategic physical effort exertion for monetary rewards. J. Exp. Psychol. Gen. 150, 103-113 (2021).

57. Mobbs, D., Trimmer, P. C., Blumstein, D. T. \& Dayan, P. Foraging for foundations in decision neuroscience: Insights from ethology. Nat. Rev. Neurosci. 19, 419-427 (2018).

58. Beck, S. M., Locke, H. S., Savine, A. C., Jimura, K. \& Braver, T. S. Primary and secondary rewards differentially modulate neural activity dynamics during working memory. PLoS One 5, (2010).

59. Levy, D. J. \& Glimcher, P. W. Comparing Apples and Oranges: Using Reward-Specific and Reward-General Subjective Value Representation in the Brain. J. Neurosci. 31, 14693-14707 (2011).

60. Yousuf, M., Heldmann, M., Göttlich, M., Münte, T. F. \& Doñamayor, N. Neural processing of food and monetary rewards is modulated by metabolic state Automatic Removal of Motion Artifacts. Brain Imaging Behav. 0, 0 (2017).

61. Odum, A. L. Delay Discounting: I'm a k, You're a k. J. Exp. Anal. Behav. 96, 427-439 (2011).

62. Westbrook, A., Kester, D. \& Braver, T. S. What Is the Subjective Cost of Cognitive Effort? Load, Trait, and Aging Effects Revealed by Economic Preference. PLoS One 8, 1-8 (2013).

63. Hart, S. G. \& Sta, L. E. [Hankock, 1988] Development of NASA-TLX_Results of Empirical and Theoretical Research. (1988).

64. Bunge Silvia A., M., D. N., E., T. M., J., V. C. \& Gabrieli D.E., J. Immature Frontal Lobe Contributions to Cognitive Control in Children: Evidence from fMRI. Neuron 33, 301-311 (2002).

65. Brydges, C. R., Anderson, M., Reid, C. L. \& Fox, A. M. Maturation of Cognitive Control: Delineating Response Inhibition and Interference Suppression. PLoS One 8, 1-8 (2013).

66. Ahn, W.-Y., Haines, N. \& Zhang, L. Revealing Neurocomputational Mechanisms of Reinforcement Learning and Decision-Making With the hBayesDM Package. Comput. Psychiatry 1, 24-57 (2017).

67. Vehtari, A., Gelman, A. \& Gabry, J. Practical Bayesian model evaluation using leave-one-out cross-validation and WAIC. Stat. Comput. 27, 1413-1432 (2017).

68. Houben, K. \& Giesen, J. C. A. H. Will work less for food: Go/No-Go training decreases the reinforcing value of high-caloric food. Appetite 130, 79-83 (2018). 
69. Inzlicht, M., Shenhav, A. \& Olivola, C. Y. The Effort Paradox: Effort Is Both Costly and Valued. Trends Cogn. Sci. 22, 337-349 (2018).

\section{Figure legends}

Figure 1. Study and task description. The study consisted of one main session with three main tasks. a) An explicit valuation task (top panel) to establish the subjective value of both reward types. b) A willingness to work task (middle panel) to measure the willingness to exert cognitive effort as a function of reward type where reward size (6 levels) and cognitive difficulty (4 levels) were parametrically varied. c) A cognitive control task (bottom panel) to measure the invigorating effect of reward type on cognitive control. In this task, participants were rewarded on a trial by trial basis for correct responses either by observing an increase in their cumulative rewards for secondary rewards or by receiving small squirts of juice using a custom-built gustometer for correct responses, as commonly done in rodent or non-human primate research. In addition to reward type, reward size (2 levels), and difficulty (2 levels) were used in this task.

Figure 2. Subjective value of reward changes across development. Individual dots depict participants, the dot plot represents the group mean with whiskers representing $95 \%$ bootstrapped confidence intervals. The green dotted line represents the grand mean across groups and conditions. a) Subjective valuation of reward types in both groups. b) Perceived worth of juice in log (pence) across both groups. 
Figure 3. Reward specific effects on exertion of cognitive effort across development. a) Average probability of accepting an offer in the cognitive effort task across cognitive difficulty levels, reward sizes and reward types for both groups. b) Discounting rate (k-parameter) from the hyperbolic model across both groups and conditions. c) Estimated cognitive load from the Nasa Task Load Index for individual difficulty levels of the control task.

Figure 4. Cognitive control is invigorated in a reward-specific way across development.

a) Participant's model coefficients from linear mixed model for both groups and reward types.

b) Correlation between participants' model coefficients and self-reported subjective value for each reinforcer. C) Participant's model coefficients from the linear mixed model for both groups and reward types on no-go trials. 


\section{Supplementary Materials}

\section{Group characteristics}

Throughout the experiment, we asked participants a series of control questions that would enable us to rule out confounds driving the observed differences reported in the manuscript.

We tested whether differences in subjective value could have arisen because adults or children were more thirsty, whether they wanted to drink juice more, and/or whether they craved it more compared to the other group. We controlled for these by adapting questions from previous work 68. We observed adults reported being more thirsty $\left(M_{\text {adults }}=68.96(3.01), M_{\text {children }}=31.41\right.$ (5.56), $\left.t_{32.43}=5.87,95 \% \mathrm{Cl}[24.52,50.58], p=<.001\right)$, wanted to drink more $\left(M_{\text {adults }}=60.92(3.85), M_{\text {children }}\right.$ $\left.=38.46(3.85), t_{37.44}=3.22,95 \% \mathrm{Cl}[8.35,36.58], p=0.002\right)$, and craved juice more $\left(M_{\text {adults }}=57.61\right.$ (4.38), $\left.M_{\text {children }}=32.14(5.04), t_{42.87}=3.72,95 \% \mathrm{Cl}[11.66,39.29], p=0.0005\right)$ compared to children. This was expected as adults were told not to drink $4 \mathrm{~h}$ before the experiment while children were not, and therefore also reported more time had passed since they last consumed any liquid $\left(t_{45.89}=3.99, p<.001\right)$. However, it is unlikely this difference would have an impact on the observed results, as the expectation would have been an opposite pattern to what we found. That is, if this had been a strong determinant of behavior, adults would prefer juice over money compared to children, which was not the case for any of the tasks measured.

\section{Willingness to work (WtW) model selection results}

The three fitted models (hyperbolic, exponential, time-sensitive) were compared using the leaveone-out-criterion (LOOIC) ${ }^{67}$ (Figure S1) which showed that across both the primary and secondary reinforcer condition, the effort-sensitive model fit both adults and children best. We formally compared per-participant model fits to investigate whether this model was consistently better across both groups in both the primary and secondary reinforcer condition.

We observed that the effort-sensitive model did not significantly better explain participants' behavior either in the primary (adults: $t_{25}=0.31, p=0.76$, children: $t_{21}=1.88, p=0.07$ ) or secondary (adults: $t_{25}=0.05, p=0.96$, children: $t_{21}=1.78, p=0.09$ ) condition in terms of the LOOIC values compared to a hyperbolic discounting model. 
Because we had no apriori hypothesis which model would fit participants best, we presented the hyperbolic model in the main text as it provides a more parsimonious explanation with one parameter compared to the effort-sensitive model with two parameters. However, we analysed the effort-sensitive model as well to see whether our results are model-specific or more general.

This analysis replicated our main findings when we compared the two parameters (discount rate, effort sensitivity) of the effort-sensitive model. The discount rate ( $r$ ) measures how strongly rewards are discounted as a function of cognitive effort that needs to be exerted to attain them. While the effort sensitivity (s) measures how discrete participants' discounting of more difficult compared to easier trials is. We found interactions between group and reward for both parameters $\left(F_{(1,92)}=\right.$ 8.01, $\left.p=0.006, F_{(1,92)}=15981.7, p<.001\right)$. Adults discounted secondary compared to primary rewards less ( $\left.t_{25}=3.8895 \% \mathrm{Cl}[0.02,0.04], p=0.0006\right)$. However, this difference was reversed in children who discounted secondary rewards more compared to primary rewards $\left(t_{21}=2.2295 \%\right.$ $\mathrm{Cl}[-0.026,-0.001], p=0.041)$. We also observed adults discounted the value of primary rewards more steeply compared to children $\left(t_{31.21}=3.2295 \% \mathrm{Cl}[0.01,0.06], p=0.003\right)$.

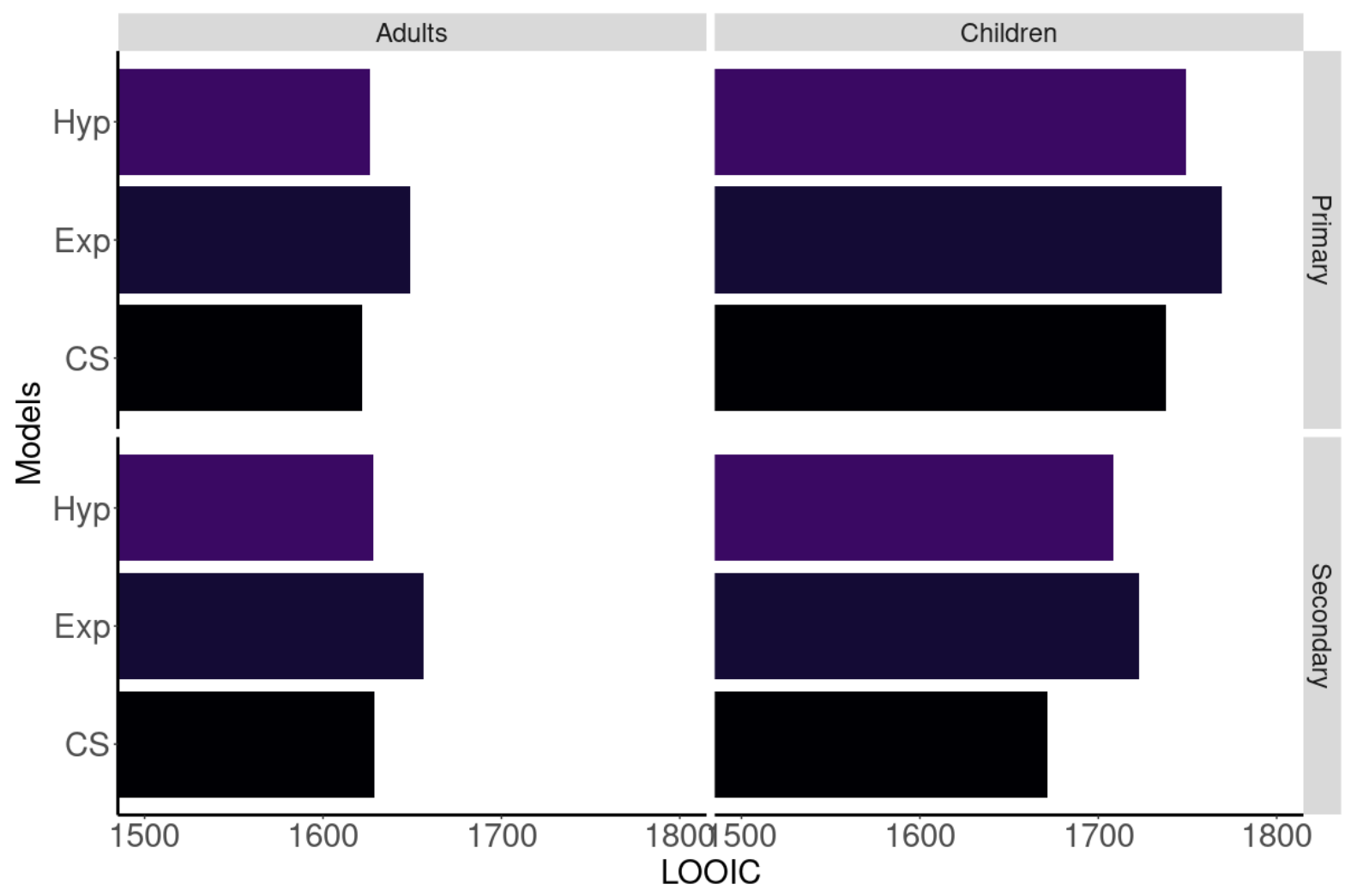


Figure S1. Leave one out criterion values for all three model types in both groups across condition. Lower values mean better model fit.

Having established that our main finding holds for the effort-sensitive model, we then examined the quality of the parameter fits of the hyperbolic model by visually investigating trace plots of posterior distributions and the $\hat{R}$ convergence scores, which indicated that across both conditions and groups all parameters converged (cognitive discounting parameter $\hat{R}$ range [0.99, 1.004]) Values close to 1 indicate good convergence.

\section{WtW supplementary task explanation}

A critical component of the willingness to work task which minimized possible confounding explanations was in the instructions. Participants were instructed they had a low chance of attaining a reward on any trial on which they would choose "yes". This was done because the expected value for trials with the highest difficulty was by definition near zero, as the probability of providing a correct answer would be at chance level, i.e.

$$
E V=R P_{\text {chance }}
$$

Where $E V$ is the expected value, $R$ is the reward on a trial, and $P_{\text {chance }}$ is the probability of providing the correct answer by chance. Therefore, they were instructed a low probability of receiving a reward, independent of their performance, existed. This meant the main reason why participants would decide not to choose "yes" would have been if they were not willing to exert cognitive effort for the task. Namely, a higher than chance level existed for obtaining a reward independent of their performance:

$$
E V=R\left(P_{\text {chance }}+P_{\text {low }}\right)
$$

Where $P_{\text {low }}$ is the low probability from the instructions given to participants.

\section{WtW control results}

We compared both groups on the need for cognition scale because we wanted to ensure that one group would not be inherently more willing to exert cognitive effort because they would find the exertion of cognitive effort rewarding in itself ${ }^{69}$. We found no difference between both groups on 
the need for cognition scale $\left(\mathrm{M}_{\text {adults }}=3.56, \mathrm{M}_{\text {children }}=3.35, t_{44.423}=1.23,95 \% \mathrm{Cl}[-0.14,0.58], \mathrm{p}=\right.$ $0.23)$.

\section{Cognitive control supplementary results}

We examined whether the effects observed on participants' probability of providing correct responses would also be observed in log reaction times using linear mixed models, but did not observe an interaction between group and reward $(p=0.15)$. However, we did find a main effect for both group $(F=4.82, p=0.028)$ and reward $(F=17.69, p<.001$ (see Supplementary Materials Table S2). 
Table 1: Results of generalized linear mixed modelling of participants' probability to provide a correct answer on go trials

\begin{tabular}{lcccc} 
Fixed Effects & Odds Ratios & Cl & z-value & $p$ \\
\hline (Intercept) & 5.91 & $4.86-7.19$ & 17.74 & $<0.001$ \\
Group & 0.54 & $0.41-0.72$ & -4.23 & $<0.001$ \\
Reinforcer & 1.34 & $1.14-1.58$ & 3.56 & $<0.001$ \\
Group * Reinforcer & 0.73 & $0.59-0.91$ & -2.85 & $\mathbf{0 . 0 0 4}$
\end{tabular}

\section{Random Effects}

\begin{tabular}{ll}
$\sigma^{2}$ & 3.29 \\
$T_{00}$ participant & 0.29 \\
$T_{11}$ Participant direction bias & 0.32 \\
$T_{11}$ Participant Trial effect & 0.03 \\
$\rho_{01}$ & -0.58 \\
& -0.07 \\
ICC & 0.08 \\
$\mathrm{~N}_{\text {sub_id }}$ & 48 \\
\hline Observations & 17127 \\
Marginal $\mathrm{R}^{2} /$ Conditional $\mathrm{R}^{2}$ & $0.042 / 0.119$
\end{tabular}


Table S2: Results of linear mixed modelling of participants' log reaction times on go trials

\begin{tabular}{|c|c|c|c|c|}
\hline Fixed Effects & Estimates & $\mathrm{Cl}$ & $t$-value & $p$ \\
\hline (Intercept) & 5.92 & $5.86-5.98$ & 199.52 & $<0.001$ \\
\hline Group & 0.10 & $0.01-0.18$ & 2.20 & 0.033 \\
\hline Reinforcer & -0.05 & $-0.07--0.03$ & -4.21 & $<0.001$ \\
\hline Group * Reinforcer & -0.03 & $-0.06-0.01$ & -1.44 & 0.158 \\
\hline \multicolumn{5}{|l|}{ Random Effects } \\
\hline$\sigma^{2}$ & 0.03 & & & \\
\hline T00 Participant & 0.03 & & & \\
\hline $\mathrm{T}_{11}$ Participant direction bias & 0.00 & & & \\
\hline $\mathrm{T}_{11}$ Participant Reinforcer effect & 0.00 & & & \\
\hline $\mathrm{T}_{11}$ Participant Trial effect & 0.00 & & & \\
\hline \multirow[t]{3}{*}{$\rho_{01}$} & -0.38 & & & \\
\hline & -0.49 & & & \\
\hline & -0.07 & & & \\
\hline ICC & 0.41 & & & \\
\hline $\mathrm{N}_{\text {Participant }}$ & 48 & & & \\
\hline Observations & 17127 & & & \\
\hline Marginal $\mathrm{R}^{2}$ / Conditional $\mathrm{R}^{2}$ & $0.046 / 0.4$ & & & \\
\hline
\end{tabular}


Table S3: Results of generalized linear mixed modelling of participants' probability of correctly withholding the prepotent response on no-go trials

\begin{tabular}{lcccc} 
Fixed Effects & Odds Ratios & $C l$ & \multicolumn{2}{l}{ Statistic $p$} \\
\hline (Intercept) & 9.88 & $6.08-16.04$ & 9.25 & $<0.001$ \\
Group & 0.23 & $0.12-0.45$ & -4.26 & $<0.001$ \\
Reinforcer & 1.41 & $1.04-1.90$ & 2.21 & $\mathbf{0 . 0 2 7}$ \\
Group * Reinforcer & 0.71 & $0.48-1.05$ & -1.71 & 0.087
\end{tabular}

\section{Random Effects}

\begin{tabular}{ll}
$\sigma^{2}$ & 3.29 \\
$T_{00}$ Participant & 1.86 \\
$\mathrm{~T}_{11}$ Participant Trial effect & 0.25 \\
$\rho_{01 \text { Participant }}$ & -0.65 \\
$\mathrm{ICC}$ & 0.36 \\
$\mathrm{~N}_{\text {Participant }}$ & 48 \\
\hline Observations & 6957 \\
Marginal $\mathrm{R}^{2}$ / Conditional $\mathrm{R}^{2}$ & $0.117 / 0.436$
\end{tabular}


Table S4: Results of generalized linear mixed modelling of participants' probability to provide a correct response on go trials

\begin{tabular}{lcccc} 
Fixed Effects & Odds Ratios & $\mathrm{Cl}$ & z-value & $p$ \\
\hline (Intercept) & 15.04 & $11.84-19.10$ & 22.22 & $<0.001$ \\
Group & 0.38 & $0.27-0.53$ & -5.72 & $<0.001$ \\
Reward size & 1.00 & $0.81-1.25$ & 0.03 & 0.973 \\
Difficulty & 0.28 & $0.23-0.33$ & -13.63 & $<0.001$ \\
Group * Reward size & 1.10 & $0.83-1.46$ & 0.66 & 0.509 \\
Group * Difficulty & 1.31 & $1.03-1.68$ & 2.20 & $\mathbf{0 . 0 2 8}$ \\
Reward * Difficulty & 1.00 & $0.77-1.30$ & 0.02 & 0.984 \\
Group * Reward size * & 0.78 & $0.55-1.10$ & -1.41 & 0.158 \\
Difficulty & & & &
\end{tabular}

\section{Random Effects}

\begin{tabular}{ll}
$\sigma^{2}$ & 3.29 \\
T00 Participant & 0.33 \\
$\mathrm{~T}_{11}$ Participant Direction bias & 0.36 \\
$\mathrm{~T}_{11}$ Participant Trial effect & 0.04 \\
$\rho_{01}$ & -0.57 \\
& 0.10 \\
$\mathrm{ICC}$ & 0.09 \\
$\mathrm{~N}$ Participant & 48 \\
\hline Observations & 17127 \\
Marginal $\mathrm{R}^{2}$ / Conditional $\mathrm{R}^{2}$ & $0.131 / 0.210$
\end{tabular}


Table S5: Results of linear mixed modelling of participants' probability to correctly withhold a response on no-go trials

\begin{tabular}{lllll} 
Fixed Effects & Odds Ratios & Cl & z-value & $p$ \\
\hline (Intercept) & 9.26 & $5.75-14.89$ & 9.17 & $<0.001$ \\
Group & 0.21 & $0.11-0.40$ & -4.70 & $<0.001$ \\
Reward size & 1.34 & $1.01-1.78$ & 2.02 & $\mathbf{0 . 0 4 3}$ \\
Difficulty & 1.32 & $0.94-1.84$ & 1.63 & 0.104 \\
Group * Reward size & 0.90 & $0.63-1.29$ & -0.56 & 0.576 \\
Group * Difficulty & 0.93 & $0.62-1.40$ & -0.34 & 0.734 \\
Reward * Difficulty & 0.81 & $0.54-1.21$ & -1.02 & 0.307 \\
$\begin{array}{l}\text { Group * Reward size * } \\
\text { Difficulty }\end{array}$ & 1.00 & $0.60-1.67$ & -0.01 & 0.993
\end{tabular}

\section{Random Effects}

\begin{tabular}{ll}
$\sigma^{2}$ & 3.29 \\
$T_{00}$ Participant & 1.61 \\
$\mathrm{~T}_{11}$ Participant Difficulty effect & 0.09 \\
$\mathrm{~T}_{11}$ Participant Trial effect & 0.28 \\
$\rho_{01}$ & 0.69 \\
& -0.62 \\
$\mathrm{ICC}$ & 0.37 \\
$\mathrm{~N}_{\text {Participant }}$ & 48 \\
\hline Observations & 6957 \\
Marginal $\mathrm{R}^{2} /$ Conditional $\mathrm{R}^{2}$ & $0.118 / 0.442$
\end{tabular}

